Chronic Obstructive Pulmonary Diseases:

Journal of the COPD Foundation

COPD

Original Research

\title{
Daily Physical Activity in Patients With COPD After Hospital Discharge in a Minority Population
}

Valentin Prieto-Centurion, MD, MS ${ }^{1}$ Richard Casaburi, PhD, MD $^{2}$ David B. Coultas, $\mathrm{MD}^{3}$

Mayank M. Kansal, MD ${ }^{1}$ Spyros Kitsiou, $\mathrm{PhD}^{1}$ Julia J. Luo, $\mathrm{BS}^{1} \mathrm{Jun}_{\mathrm{Ma}} \mathrm{MD}, \mathrm{PhD}^{1}$ Cynthia S. Rand, $\mathrm{PhD}^{4}$

Ai-Yui M. Tan, $\mathrm{MD}^{1}$ Jerry A. Krishnan, MD, $\mathrm{PhD}^{1,5}$

\section{Abstract}

Background: Low physical activity in patients with chronic obstructive pulmonary disease (COPD) is associated with increased morbidity and mortality. To inform the design of a home-based physical activity promotion program for patients with COPD recently discharged from a minority-serving hospital, we conducted a cohort study to evaluate objectively measured daily physical activity and patient-reported outcomes.

Methods: This was a 12-week prospective cohort study of patients with a physician diagnosis of COPD recently hospitalized ( $\leq 12$ weeks) for respiratory symptoms. Daily physical activity was recorded using wrist-based and "clip-on" pedometers, and analyzed as mean daily step counts averaged over 7 days.

Results: Twenty-two patients were enrolled a median (interquartile range, [IQR]) of 14 (7 to 29) days after hospital discharge. The median daily step count (IQR) in the first week after enrollment (week 1) was 3710 (1565 to 5129) steps. The median within-person change in daily step count (IQR) from week 1 to week 12 was 314 (-30 to 858 ) steps ( $p=0.28$ ). Within-person correlation of week-to-week daily step counts was high $(r \geq 0.75)$. Time from hospital discharge to enrollment was not correlated with mean daily step counts on week $1(\mathrm{r}=-0.13)$ and only weakly correlated with change in mean daily step counts from week 1 to week 12 ( $\mathrm{r}=0.37)$.

Conclusions: Daily physical activity was variable in this cohort of recently hospitalized patients with COPD, but with little within-person change over a 12-week period. These observations highlight the need for flexible physical activity promotion programs addressing the needs of a heterogeneous patient population.

Abbreviations: chronic obstructive pulmonary disease, COPD; interquartile range, IQR; incremental shuttle walk test, ISWT; PatientReported Outcomes Measurement System, PROMIS; standard deviation, SD; body mass index, BMI; kilograms per square meter, $\mathrm{kg} / \mathrm{m}^{2}$; forced expiratory volume in 1 second, $\mathbf{F E V}_{\mathbf{1}}$; number of participants with missing information, $\mathbf{m}$

Funding Support: Dr. Prieto-Centurion was funded by National Institutes of Health grants (K23HL130524 and R25HL126146).

Date of Acceptance: September 13, 2019

Citation: Prieto-Centurion V, Casaburi R, Coultas DB, et al. Daily physical activity in patients with COPD after hospital discharge in a minority population. Chronic Obstr Pulm Dis. 2019;6(4):332-340. doi: https://doi.org/10.15326/jcopdf.6.4.2019.0136

\section{University of Illinois at Chicago}

2 Los Angeles Biomedical Research Institute at Harbor-University of California-Los Angeles Medical Center, Torrance

3 Portland VA Medical Center, Portland, Oregon

4 Johns Hopkins University, Baltimore, Maryland

5 Institute for Healthcare Delivery Design, University of Illinois Hospital and Health Sciences System, Chicago

\author{
Address correspondence to: \\ Valentin Prieto-Centurion, MD, MS \\ University of Illinois at Chicago \\ 1220 S. Wood St, MC 619 \\ Chicago, IL 60608 \\ Phone: (312) 413-1960 \\ Email: vprieto@uic.edu
}

\section{Keywords:}

physical activity; COPD; post-hospital discharge 


\section{Introduction}

Low physical activity is common in patients with chronic obstructive pulmonary disease (COPD) and is associated with increased morbidity and mortality. ${ }^{1-3}$ Pulmonary rehabilitation after hospital discharge has been shown to improve multiple outcomes in patients with COPD, but its impact is limited due to low levels of participation. ${ }^{4,5}$ A recent study of Medicare patients found that less than $3 \%$ of patients start pulmonary rehabilitation within 12 months of hospital discharge and that approximately $90 \%$ of them do not complete it. ${ }^{6}$ Limited availability of pulmonary rehabilitation programs, as well as transportation and financial difficulties have been identified as common barriers. 7,8 Minority and low-income patients with COPD have more barriers to participation in pulmonary rehabilitation programs and have a higher likelihood of poor outcomes after hospital discharge. ${ }^{9,10}$ Physical activity promotion interventions may be feasible and pragmatic for underserved patients with COPD recently discharged from the hospital, but evidence for their effectiveness is lacking. To inform the design of a home-based physical activity promotion program for patients with COPD recently discharged from a minority-serving hospital, we conducted a cohort study to evaluate objectively measured levels of daily physical activity and patient-reported outcome.

\section{Methods}

This was a 12-week, prospective cohort study of patients with a physician diagnosis of COPD recently hospitalized ( $\leq 12$ weeks since hospital discharge) for respiratory symptoms at an academic medical center and minority-serving institution located in Chicago, Illinois. As less than $2 \%$ of patients with COPD enroll in pulmonary rehabilitation after hospital discharge, we selected a 12-week enrollment window after hospital discharge to identify a feasible timeframe for participants to initiate a home-based physical activity promotion program. ${ }^{6}$ Additional eligibility criteria included physical ability to walk and no medical contraindications to participation, as determined by the patient's physician. Electronic health records were screened to identify hospitalized patients with a diagnosis of COPD or patients attending the outpatient clinics who had been recently hospitalized.
Written informed consent was obtained from all study participants. The study was approved by the Institutional Review Board at the University of Illinois at Chicago.

Daily physical activity was recorded over a 12week period using either wrist-based (Fitbit Charge HR) or "clip-on" (Fitbit Zip) Bluetooth-enabled pedometers, which allow remote physical activity data collection. ${ }^{11,12} \mathrm{Clip}$-on pedometers were preferentially used for patients in whom there was a concern about accuracy of wrist-based pedometers (e.g., use of a rolling walker that limited hand motion during normal walking). ${ }^{13}$ Daily physical activity was measured as the mean daily step counts over each 7 -day period. At least 4 days per week with wearing time $\geq 8$ hours were required for a valid assessment. ${ }^{14}$ Presence of heart rate data and intra-day time gaps between steps was used to calculate the pedometer wear time.

The incremental shuttle walk test (ISWT), a measure of maximal functional exercise capacity, was performed at the enrollment visit. ${ }^{15}$ Participants also reported on their health care utilization (i.e., allcause hospitalizations) and multiple domains of health using the Patient-Reported Outcomes Measurement Information System (PROMIS), including physical function, fatigue, anxiety, depression, ability to participate in social roles and activities, and satisfaction with social roles and activities. ${ }^{16}$ PROMIS scores are standardized such that the U. S. national population has a mean T-score of 50 units with a standard deviation (SD) of 10 units. Higher T-scores indicate more of the concept being measured, i.e., a higher PROMIS fatigue T-score indicates worse fatigue whereas a higher PROMIS physical function T-score indicates better physical function. A difference in T-scores of $\geq 5$ units (i.e., 0.5 SD) was considered clinically meaningful. ${ }^{17}$ Pearson's $r$ was used to establish correlations between mean daily step counts and time from hospital discharge to study enrollment. Wilcoxon rank sum tests were used to compare differences in mean daily step counts according to health care utilization and functional capacity. Wilcoxon signed rank tests were used to compare time-related changes in patientreported outcomes. Analyses were performed using STATA software package, release 15.1 (Stata Corp Inc, College Station, Texas). 


\section{Results}

Twenty-two patients were enrolled at a median (interquartile range, [IQR]) of 14 (7 to 29) days after hospital discharge (Table 1). Participants had a mean (SD) age of 63.8 (7.1) years, were predominantly women (68\%), African American (91\%), and had no more than a high school education (72\%). Nine participants were rehospitalized and 3 died during the follow-up period. Comorbidities were common among the participants, including those associated with chronic pain (45\% reported connective tissue disease, osteoarthritis or chronic back pain) or limited mobility (23\% reported a previous stroke). Additionally, some of the comorbidities reported by participants have symptoms that can either overlap with or exacerbate COPD (e.g., heart failure, perception of dyspnea in patients with comorbid depression). Participants had a median (IQR) ISWT distance of 90 (25 to 205) meters. On enrollment, the majority of participants reported physical function (77\%), ability to participate in social roles and activities (77\%), and satisfaction with social roles and activities (72\%) T-scores significantly lower than the U.S. general population.

The median daily step count (IQR) in the first week after enrollment (week 1) was 3710 (1565 to 5129) steps. The median within-person change in daily step count (IQR) from week 1 to week 12 was 314 (-30 to $858)$ steps $(p=0.28)$. In addition, the within-person correlation of week-to-week daily step counts was high $(r \geq 0.75)$, indicating limited variability in daily physical activity over the 12 weeks of observation. Time from hospital discharge to enrollment was not correlated with mean daily step counts on week 1 ( $\mathrm{r}=$ -0.13; Figure 1, Panel A) and only weakly correlated with the change in mean daily step counts from week 1 to week 12 ( $r=0.37$; Figure 1, Panel B).

Mean daily step counts during week 1 were significantly lower among participants with ISWT distances below the median value than those with distances above the median (1230 versus 4459 steps per week, $p=0.02$; Figure 2, Panel A ). Mean daily step counts during week 1 were also lower among participants who were rehospitalized or died during the study period than those who were not, although this difference was not statistically significant (3660 versus 4864 steps per week, $p=0.26$; Figure 2, Panel B). Compared to week 1 , there was no significant difference in any of the PROMIS domains T-scores at week 12 ( $p>0.05$ for all measures; Figure 3).

\section{Discussion}

In predominantly African American patients with COPD, recently discharged from the hospital, we observed a wide range of mean daily step counts, but without significant within-person change over the 12-week observation period. These patients had a high rate of poor clinical outcomes following hospital discharge. In addition, they had low functional exercise capacity as measured by the ISWT (ageadjusted normal ISWT distance for 60-69-year-old individuals is $788 \mathrm{~m}) .{ }^{18}$ The observed mean daily step counts and ISWT distance was more than the minimal clinical important difference (1100 steps per day and 47.5 meters, respectively) and lower than that observed in previous studies of patients with stable COPD. ${ }^{19-22}$ A high proportion of participants also reported limited physical or social health, as recorded by several PROMIS domains, without any significant change over the observation period. We did find an association between higher ISWT distance and higher mean daily step counts. This observation is consistent with the concept that physical conditioning, a physiologic characteristic, is a significant determinant of a behavior, daily physical activity, in this population.

These observations have implications for the design of programs to improve physical activity and outcomes following hospital discharge in patients with COPD. First, although these patients have variable levels of physical activity after hospital discharge, a large majority of patients reported limited physical and social functioning. Therefore, to be responsive to the needs of these patients, physical activity promotion programs need to be flexible to allow participation of patients with markedly different levels of physical activity on enrollment. Second, given the high prevalence of comorbidities and high baseline risk of poor clinical outcomes in this population, close collaboration with the patients' health care providers is essential to address any potential changes in clinical status. Third, as we did not observe a significant association between daily physical activity and the time from hospital discharge to enrollment, patients who are beyond the immediate post-hospital discharge period may still benefit from physical activity promotion programs. The immediate post-hospital discharge period can be chaotic for many 


\section{Table 1. Participant Characteristics on Study Enrollment}

Characteristic

\section{Demographic}

Age (years), mean (SD)

Female Gender, $\mathrm{n}(\%)$

African American Race, $\mathrm{n}(\%)$

High School Education or Less, $\mathrm{n}(\%)$

Current Smoker, n (\%)

Clinical

BMI $\left(\mathrm{kg} / \mathrm{m}^{2}\right)$, median (IQR), $\mathrm{m}=2$

$\%$ Predicted $\mathrm{FEV}_{1}$, mean (SD), $\mathrm{m}=2$

Hospitalizations in Previous Year, mean (SD)

ISWT Distance $(\mathrm{m})$, median (interquartile range), $\mathrm{m}=3$

Week 1 Daily Step Counts, median (IQR), $m=4$

Comorbidities, $\mathrm{n}(\%)$

Hypertension

Diabetes

Sleep Apnea

Heart Failure

Chronic Back Pain

Connective Tissue Disease

Stroke

Depression

Osteoarthritis

Week 1 PROMIS Domains, $\geq 5$-unit difference from $50^{\mathrm{a}}$

Anxiety, $\mathrm{n}(\%), \mathrm{m}=1$

Depression, $\mathrm{n}(\%)$

Fatigue, $\mathrm{n}(\%), \mathrm{m}=1$

Physical Function, $\mathrm{n}(\%)$

Ability to Participate in Social Roles \& Activities, n (\%)

Satisfaction with Participation in Social Roles \& Activities, $n(\%), m=1$
Baseline measure, $\mathrm{n}=22$

$63.8(7.1)$

15 (68\%)

20 (91\%)

$16(72 \%)$

$5(23 \%)$

32.5 (27.1 to 41.0$)$

$52(15.0) \%$

$1.8(1.1)$

90 (25 to 205)

3710 (1565 to 5129$)$

$\mathrm{SD}=$ standard deviation; $\mathrm{BMI}=$ body mass index; $\mathrm{kg} / \mathrm{m}^{2}=$ kilograms per square meter; IQR=interquartile range; $\mathrm{m}=$ number of participants with missing information; $\mathrm{FEV}_{1}=$ forced expiratory volume in 1 second; ISWT=incremental shuttle walk test; PROMIS=Patient-Reported Outcomes Measurement Information System

${ }^{a} A$ difference in T-scores of $\geq 5$ units $(0.5 \mathrm{SD})$ from the U.S. general population mean ( 50 units) is considered clinically meaningful. Higher T-scores indicate more of the concept being measured.

patients and their caregivers as they attempt to return to their normal routine. ${ }^{23}$ Additionally, although the risk for rehospitalization or death is highest within the first weeks after hospital discharge, this risk remains elevated for at least 1 year. $^{24}$ Flexible timelines for enrollment in post-hospitalization physical activity promotion programs may allow inclusion of patients who otherwise may not have been able to participate. Third, although ISWT distance allows insight into the mechanisms by which a physical activity promotion program works, its implementation in clinical studies adds significant burden as participants usually need to attend in-person study visits for outcome collection. As mean daily step counts, remotely collected by wireless pedometers, were significantly associated with ISWT distance, they could serve as a behavioral marker of intervention progress.

The principal strength of this study is that we used validated and scalable measures to concurrently collect data on functional capacity, daily physical activity and patient-reported outcomes in a population at high risk for adverse outcomes. The study's main limitation 
Figure 1. Mean Daily Step Count on the Week After Enrollment (A) and Change in Mean Daily Step Counts Over the Observation Period (B) According To Time From Hospital Discharge To Enrollment

A

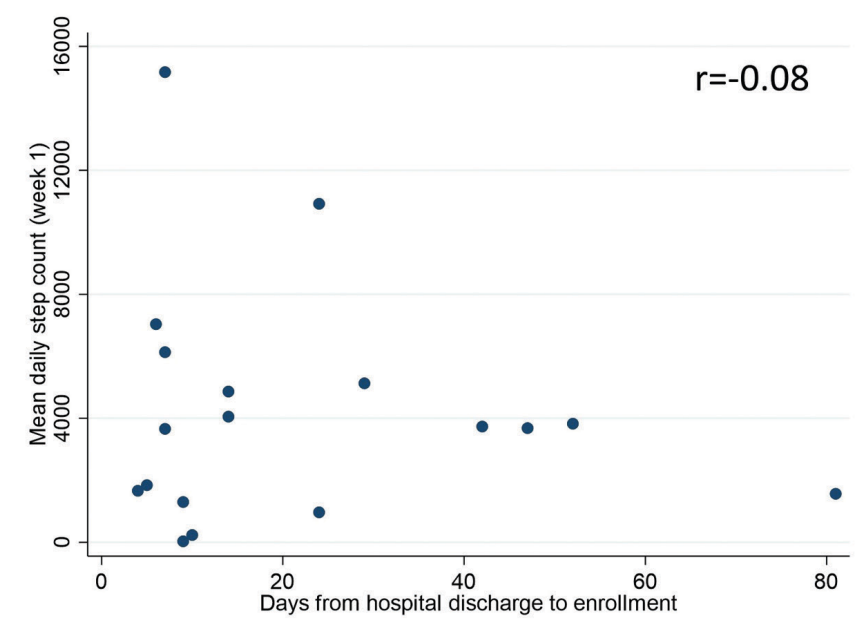

B

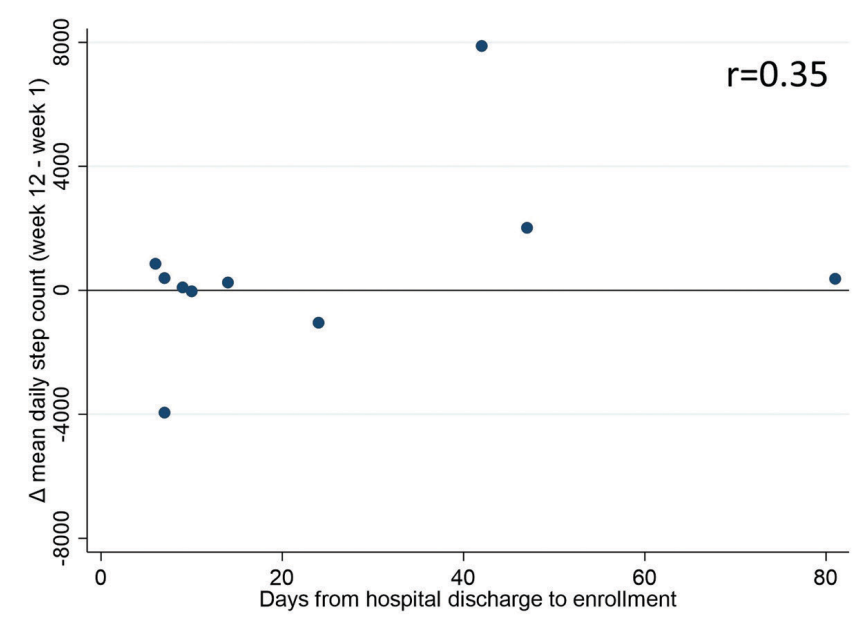

Figure 2. Mean Daily Step Count During the Week After Enrollment ${ }^{a}$ According to the Baseline Incremental Shuttle Walk Distance (A), or Whether They Were Hospitalized or Died During the Observation Period (B)

A

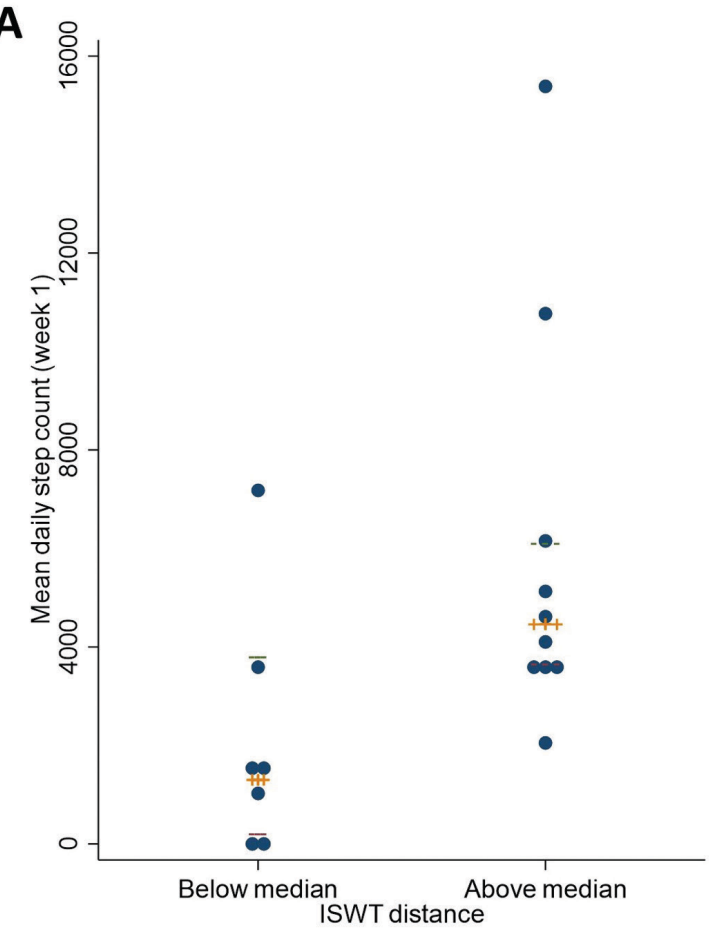

B

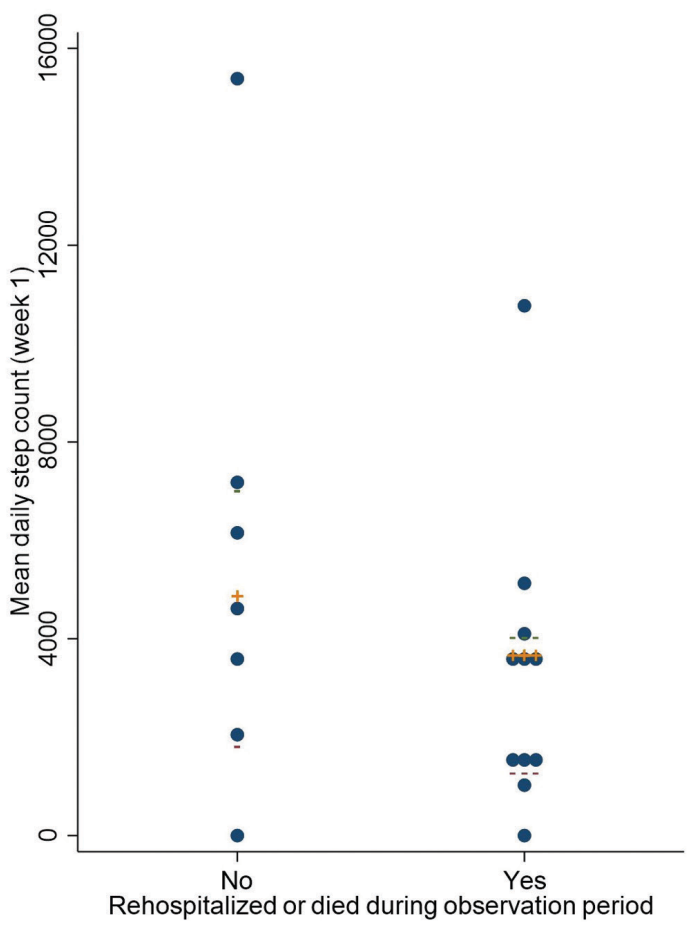

aWeek 1 


\section{Figure 3. PROMIS Domains T-Scores on Study Enrollment ${ }^{\mathrm{a}}$ and Completion $^{\mathrm{b}}$}

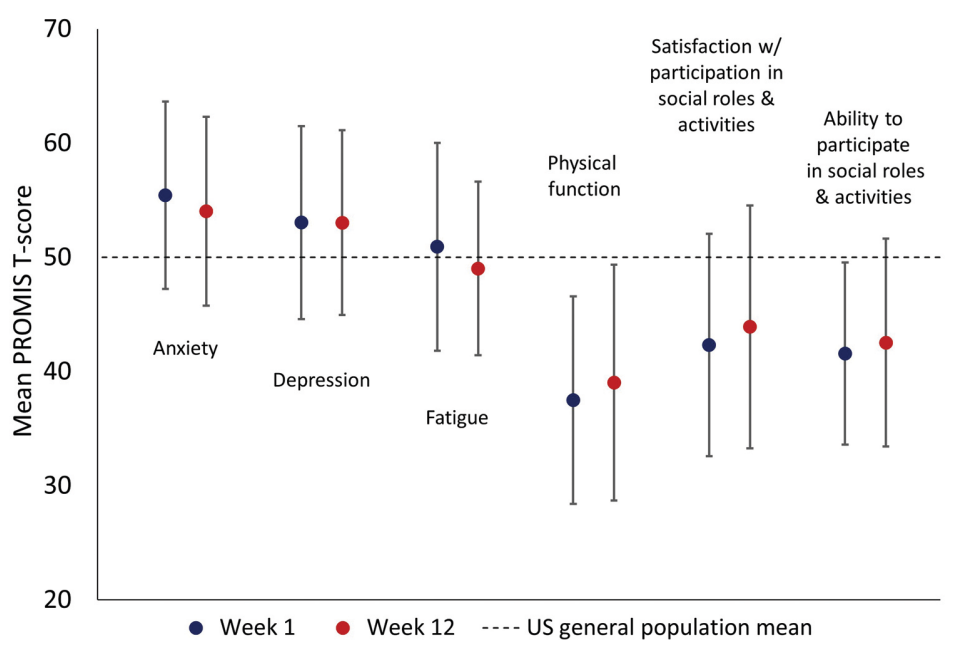

\footnotetext{
aWeek 1

${ }^{b}$ Week 12

$p>0.05$ for comparisons between week 1 and week 12 for all domains
}

PROMIS=Patient-Reported Outcomes Measurement Information System

is the relatively small sample size. It is possible that a larger sample size would have allowed us to detect differences in daily physical activity in sub-groups of patients, such as those likely to be rehospitalized during the observation period, or to characterize the study population in more detail, such as identifying comorbidities associated with lower levels of physical activity. However, the sample size is within the generally recommended number of participants for early pilot studies and it provides important feasibility data for the design of physical activity promotion programs specific to this population. ${ }^{25,26}$ Another study limitation is that, as described in the methods section, participants in whom there was a concern about the accuracy of wrist-based pedometers were provided with clip-on pedometers. A recent systematic review found that Fitbit devices worn on the wrist or torso can, in the majority of cases, provide step count measurements comparable to research-grade accelerometers or pedometers. However, it is possible that between-person differences in mean daily step counts observed in the present study are partly due to differential accuracy between pedometers. ${ }^{27}$ Last, observations were conducted in patients discharged from a single minority-serving institution and may not be applicable to other populations of patients with COPD. However, this represents the first such characterization of an underserved and relatively understudied population. Additional studies would be needed to establish the baseline characteristics of other populations to design physical activity promotion programs targeted to their needs.

In conclusion, this cohort study showed daily physical activity levels to be highly variable among patients with COPD recently discharged from the hospital, but with little within-person change over a 12-week period. These observations highlight the need for the development of flexible physical activity promotion programs that can address the needs of a heterogeneous patient population.

\section{Acknowledgements}

We thank the patients and clinicians who assisted in the implementation of the study. We also thank members of the independent Data and Safety Monitoring Board (Sherita Chapman-Smith, MD; Kerri Morgan, PhD, and Meghan Lane-Falls, MD, MSHP).

Author Contributions: VP had full access to all data in the study and takes responsibility for the integrity of the data and the accuracy of the data analysis. VP contributed to the study conception, data acquisition, 
analysis and interpretation, writing and revision of the manuscript. RC, DBC, MK, JM, and CSR contributed to the study conception, data interpretation, writing and revision of the manuscript. JJL and AMT contributed to the data acquisition and analysis, writing and revision of the manuscript. JK contributed to the study conception, data analysis and interpretation, writing and revision of the manuscript.

\section{Declaration of Interest}

Dr. Prieto-Centurion reports receiving grants from the National Institutes of Health/National Heart, Lung, and Blood Institute during the time of this study. Dr. Krishnan reports grants from the National Institutes of Health/Patient-Centered Outcomes Research Institute not related to this study and Dr. Tan reports a grant from the National Institutes of Health. All other authors have nothing to declare. 


\section{References}

1. Spruit MA, Pitta F, McAuley E, ZuWallack RL, Nici L. pulmonary rehabilitation and physical activity in patients with chronic obstructive pulmonary disease. Am J Respir Crit Care Med. 2015; 192(8):924-933.

doi: https://doi.org/10.1164/rccm.201505-0929CI

2. Watz H, Pitta F, Rochester CL, et al. An official European Respiratory Society statement on physical activity in COPD. Eur Respir J. 2014;44(6):1521-1537.

doi: https://doi.org/10.1183/09031936.00046814

3. Nguyen HQ, Chu L, Liu IL, et al. Associations between physical activity and 30-day readmission risk in chronic obstructive pulmonary disease. Ann Am Thorac Soc. 2014;11(5):695-705. doi: https://doi.org/10.1513/AnnalsATS.201401-017OC

4. Puhan MA, Gimeno-Santos E, Cates CJ, Troosters T. Pulmonary rehabilitation following exacerbations of chronic obstructive pulmonary disease. Cochrane Database Syst Rev. 2016;12:CD005305.

doi: https://doi.org/10.1002/14651858.CD005305.pub4

5. Nishi SP, Zhang W, Kuo YF, Sharma G. Pulmonary rehabilitation utilization in older adults with chronic obstructive pulmonary disease, 2003 to 2012. J Cardiopulm Rehabil Prev. 2016;36:375382. doi: https://doi.org/10.1097/HCR.0000000000000194

6. Spitzer KA, Stefan MS, Priya A, et al. Participation in pulmonary rehabilitation after hospitalization for chronic obstructive pulmonary disease among Medicare beneficiaries. Ann Am Thorac Soc. 2019;16(1):99-106. doi: https://doi.org/10.1513/AnnalsATS.201805-332OC

7. Thorpe O, Kumar S, Johnston K. Barriers to and enablers of physical activity in patients with COPD following a hospital admission: a qualitative study. Int J Chron Obstruct Pulmon Dis. 2014;9:115-128. doi: https://doi.org/10.2147/COPD.S54457

8. Benzo R, Wetzstein M, Neuenfeldt P, McEvoy C. Implementation of physical activity programs after COPD hospitalizations: lessons from a randomized study. Chron Respir Dis. 2015;12(1):510. doi: https://doi.org/10.1177/1479972314562208

9. Williams DR, Priest N, Anderson NB. Understanding associations among race, socioeconomic status, and health: patterns and prospects. Health Psychol. 2016;35(4):407-411. doi: https://doi.org/10.1037/hea0000242

10. Elixhauser A, Au DH, Podulka J. Readmissions for chronic obstructive pulmonary disease, 2008. HCUP Statistical Brief \#121. Healthcare Cost and Utilization Project website. http:// www.hcup-us.ahrq.gov/reports/statbriefs/sb121.jsp

Accessed April 6, 2019. Published 2011.

11. Fitbit Zip Specs. Fitbit website. https://www.fitbit.com/zip/specs. Published 2015. Updated 2019. Accessed January 19, 2015.
12. Fitbit Charge HR Specs. Fitbit website. https://www.fitbit.com/ chargehr\#specs

Published 2015. Updated 2019. Accessed December 29, 2015

13. Floegel TA, Florez-Pregonero A, Hekler EB, Buman MP. Validation of consumer-based hip and wrist activity monitors in older adults with varied ambulatory abilities. J Gerontol A Biol Sci Med Sci. 2016;72(2):229-236. doi: https://doi.org/10.1093/gerona/glw098

14. Demeyer H, Burtin C, Van Remoortel H, et al. Standardizing the analysis of physical activity in patients with COPD following a pulmonary rehabilitation program. Chest. 2014; 146(2):318-327. doi: https://doi.org/10.1378/chest.13-1968

15. Holland AE, Spruit MA, Troosters T, et al. An official European Respiratory Society/American Thoracic Society technical standard: field walking tests in chronic respiratory disease. Eur Respir J. 2014;44(6):1428-1446. doi: https://doi.org/10.1183/09031936.00150314

16. Patient-Reported Outcomes Measurement Information System (PROMIS). HealthMeasures website. http://www. healthmeasures.net/explore-measurement-systems/promis Published 2015. Updated 2019. Accessed February 2, 2015.

17. Health Measures. Meaningful change for PROMIS. HealthMeasures website. http://www.healthmeasures.net/scoreand-interpret/interpret-scores/meaningful-change Published 2019. Accessed April 8, 2019

18. Harrison SL, Greening NJ, Houchen-Wolloff L, et al. Age-specific normal values for the incremental shuttle walk test in a healthy British population. J Cardiopulm Rehabil Prev. 2013;33(5):309313. doi: https://doi.org/10.1097/HCR.0b013e3182a0297e

19. Moy ML, Danilack VA, Weston NA, Garshick E. Daily step counts in a US cohort with COPD. Respir Med. 2012;106(7):962969. doi: https://doi.org/10.1016/j.rmed.2012.03.016

20. McKeough Z, Leung R, Neo JH, et al. Shuttle walk tests in people with COPD who demonstrate exercise-induced oxygen desaturation: an analysis of test repeatability and cardiorespiratory responses. Chron Respir Dis. 2018;15(2):131137. doi: https://doi.org/10.1177/1479972317729051

21. Singh SJ, Jones PW, Evans R, Morgan MD. Minimum clinically important improvement for the incremental shuttle walking test. Thorax. 2008;63(9):775-777. doi: https://doi.org/10.1136/thx.2007.081208

22. Demeyer H, Burtin C, Hornikx M, et al. The minimal important difference in physical activity in patients with COPD. PLoS One. 2016;11:e0154587.

doi: https://doi.org/10.1371/journal.pone.0154587

23. Ursan ID, Krishnan JA, Pickard AS, et al. Engaging patients and caregivers to design transitional care management services at a minority serving institution. J Health Care Poor Underserved. 2016;27(1):352-365. doi: https://doi.org/10.1353/hpu.2016.0026 
24. Lindenauer PK, Dharmarajan K, Qin L, Lin Z, Gershon AS, Krumholz HM. Risk trajectories of readmission and death in the first year after hospitalization for chronic obstructive pulmonary disease. Am J Respir Crit Care Med. 2018;197(8):1009-1017. doi: https://doi.org/10.1164/rccm.201709-1852OC

25. Birkett MA, Day SJ. Internal pilot studies for estimating sample size. Stat Med. 1994;13(23-24):2455-2463.

doi: https://doi.org/10.1002/sim.4780132309

26. Julious SA. Sample size of 12 per group rule of thumb for a pilot study. Pharm Stat. 2005;4(4):287-291.

doi: https://doi.org/10.1002/pst.185

27. Feehan LM, Geldman J, Sayre EC, et al. Accuracy of Fitbit devices: systematic review and narrative syntheses of quantitative data. JMIR Mhealth Uhealth. 2018;6(8):e10527.

doi: https://doi.org/10.2196/10527 